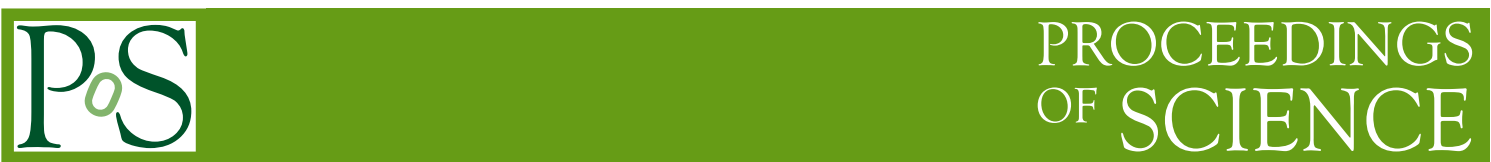

\title{
The Angstrom Survey: Status Report
}

\section{Matthew J. Darnley*}

Astrophysics Reseach Institute, Liverpool John Moores University, UK

E-mail: mjdeastro.livjm.ac.uk

The Angstrom Survey is undertaking an optical survey of stellar microlensing events across the bulge region of the Andromeda Galaxy (M31) using a distributed network of $2 \mathrm{~m}$ class telescopes. The Angstrom Project Alert System (APAS) has been developed to identify candidate microlensing and transient events in real-time, using data from the fully robotic Liverpool and Faulkes North telescopes. Here we briefly describe the Angstrom data reduction pipeline and the APAS. We present a few example lightcurves obtained during the APAS commissioning phase that clearly demonstrate its real-time capability to identify microlensing candidates as well as other transient sources.

The Manchester Microlensing Conference: The 12th International Conference and ANGLES Microlensing Workshop

January 21-25 2008

Manchester, $U K$

\footnotetext{
${ }^{*}$ Speaker.
} 


\section{The Angstrom project}

The Andromeda Galaxy Stellar Robotic Microlensing (Angstrom) project is a high cadence, optical, pixel-microlensing survey of the bulge of the Andromeda Galaxy [1]. The main aim of the Angstrom Project is to probe the geometry of the underlying mass distribution of the M31 bulge by searching for gravitational microlensing signatures from low mass stars. We also aim to characterise the mass function of low mass stars within the M31 bulge.

M31 presents a challenging target for real-time microlensing discovery. At a distance of 780 $\mathrm{kpc}$ it is two orders of magnitude more distant than typical microlensing sources towards the Galactic bulge. As such, larger telescopes and longer exposures are both required to obtain sufficiently sensitive photometry. Is also means that we have to deal with stellar fields which are far more crowded and where the typical microlensing source is completely unresolved at baseline (see Figure 1), the so-called pixel-microlensing regime.

Difference imaging techniques are required to allow such events to be detected reliably. What one effectively observes is the peak of the underlying transient flux variation and this typically lasts only a few days for bulge microlensing involving low mass stars. Because of this, the bulge must be observed many times per night and this necessitates the use of several telescopes at widely separated longitudes to allow 24-hour surveillance.

The Angstrom project employs a distributed network of $2 \mathrm{~m}$-class telescopes. These include the fully robotic $2 \mathrm{~m}$ Liverpool Telescope (LT [2]) on La Palma and 2m Faulkes Telescope North (FTN) in Hawaii, the $1.8 \mathrm{~m}$ telescope at Bohyunsan Observatory in Korea, the $2.4 \mathrm{~m}$ telescope at MDM in Arizona and the $1.5 \mathrm{~m}$ at Maidanak Observatory in Uzbekistan.

This high cadence strategy should also allow exotic microlensing phenomena to be detected with greater efficiency $[3,4]$, and will provide a new window on other transient sources or variable stars which may vary on very short timescales.
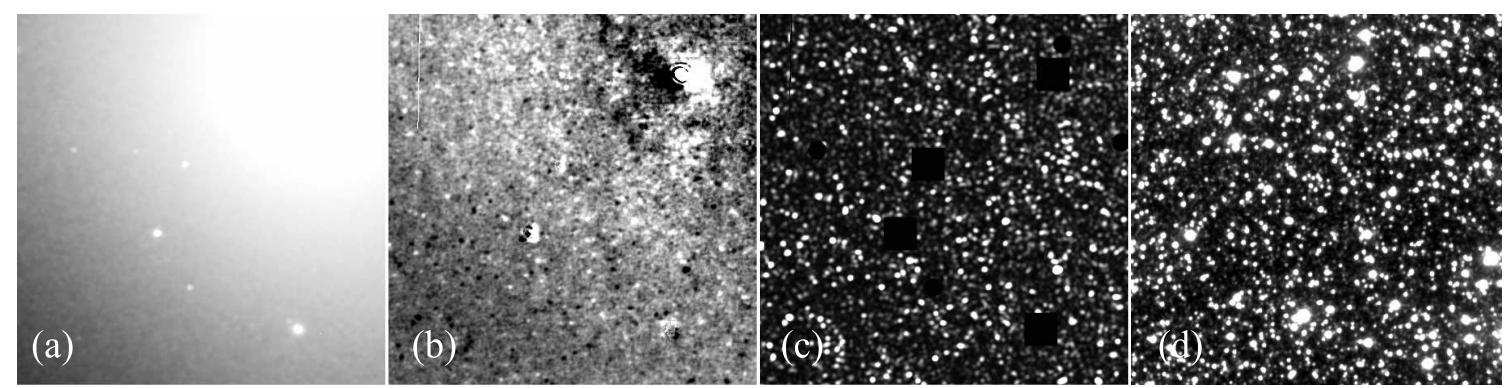

Figure 1: (a) LT $i^{\prime}$-band image of a 2' region of the Angstrom M31 bulge field. (b) Corresponding difference image obtained after convolving and subtracting a reference image, showing variable objects as white and black spots. The residual of the imperfectly subtracted core of the M31 bulge is visible in the top right-hand corner. (c) The corresponding significance map of variable sources visible above the background. Variable source blending is evident across the map. (d) Stars within a 2' region of an OGLE-III Milky Way bulge field shown for comparison with the crowding levels seen in the variable source map in (c). While all objects in (c) have variable flux, only a handful of the stars visible in (d) are variables. OGLE image is from the OGLE Early Warning System [5] for alert OGLE-2005-BLG-172. 


\section{Data pipeline}

The Angstrom Data Analysis Pipeline (ADAP) is responsible for reducing all Angstrom data from both robotic and non-robotic telescopes, The nominal mode of operation is to process the full data-set offline at the end of each observing season. However, we also employ the ADAP to process a lower resolution copy of the robotic telescope image that can be processed in real-time.

\subsection{Difference imaging}

Pixel lensing techniques require very accurate relative photometry, as such any seeing or background light variations between epochs can be problematic. Image matching techniques, such as difference image analysis (DIA) can be employed to solve these problems.

The ADAP uses a modified variant of the ISIS [6] image reduction package. This modified version, currently dubbed "AngstromISIS", has been stripped down to act solely as an efficient difference image engine.

\subsection{Source detection \& photometry}

The essential method for the difference image source detection is based upon a modified version of the Cash Statistic [7]. At each pixel the "Cash-like" statistic is determined by allowing the effective flux of the PSF to vary and then also by fixing the flux to zero. The difference between these two values has properties very similar to $\Delta \chi^{2}$ and so a "likelihood map" (see Figure 1) can be created. In addition a best-fit pixel flux map is created. Any peaks above a given threshold in the likelihood map are found and the position centroided. The flux at each centroided position is then determined using profile-fitting photometry, giving both the position and flux of the object. A MySQL database is used to efficiently manage all the detected objects from every epoch.

\section{Angstrom real-time alert system}

The Angstrom Project Alert System (APAS [8]) was commissioned during the beginning of the third Angstrom observing season (06B). By the end of that season it had enabled us to discover a very short time-scale microlensing candidate and to produce one of the best-sampled extragalactic classical nova light curves (see Figure 2).

APAS was developed to identify candidate microlensing and transient events in real-time, using data from the robotic LT and FTN. This is the first time that real-time microlensing discovery has been attempted outside the Milky Way and its satellite galaxies. The APAS is designed to enable follow-up studies of M31 microlensing systems, including searches for gas giant planets in M31.

In addition to LT and FTN data, the APAS uses four seasons of archive data from the POINTAGAPE dark matter microlensing survey of M31 [9]. Around 65\% of the LT/FTN field overlaps with the POINT-AGAPE data, which is used to provide an extended data baseline for the subset of objects lying in both survey regions.

APAS has been fully operational throughout the ongoing fourth observing season (07B). However, due to a number of technical problems with the robotic telescopes and the pipeline, we were not able to utilise the full potential of this system. Despite these problems, we have detected a 

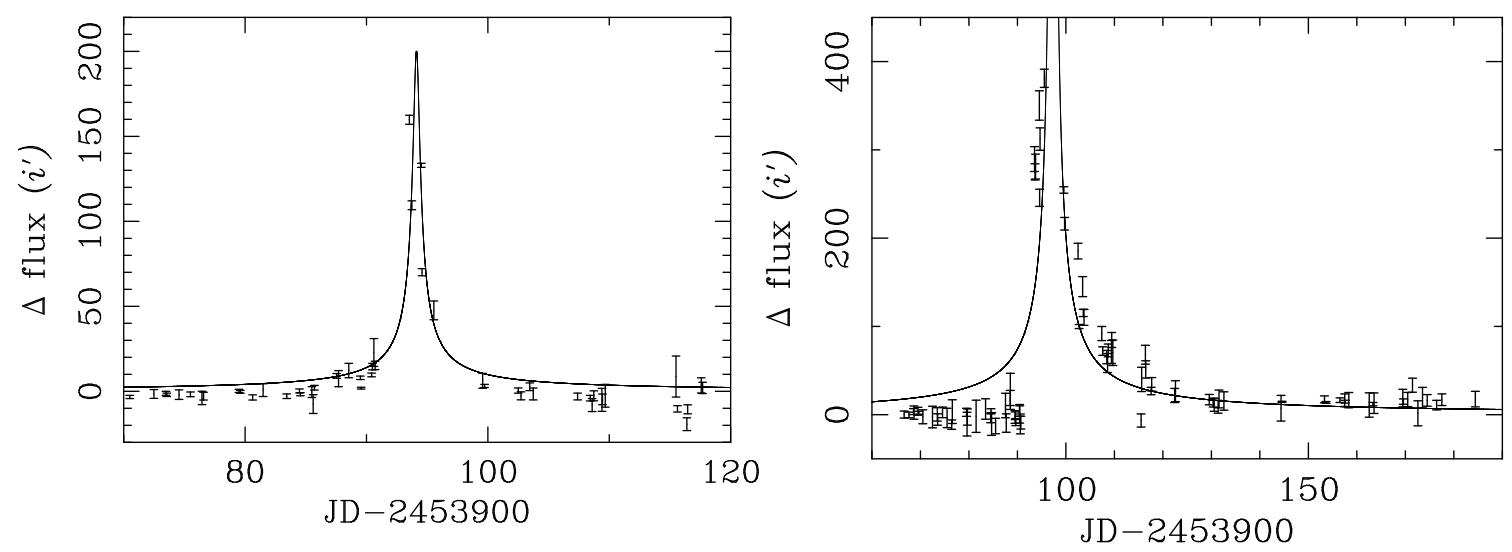

Figure 2: Two examples of alerts selected by APAS using LT data. (Left) A short timescale $\left(t_{\mathrm{FWHM}} \simeq 1\right.$ ) high signal-to-noise ratio microlensing candidate. (Right) Classical Nova M31 2006-8. This nova has a decline time of $t_{2}<10$ days, making it among the best-sampled extragalactic "very fast" CNe.

number of promising microlensing candidates during the fourth season. We have also been able to identify a nova extremely close to the centre of M31 [10].

\section{The Future}

Following the completion of this observing season we intend to re-analyse the entire four years of data from our five telescopes, including the additional four years of overlapping POINT-AGAPE data.

Our shorter term aims also include the production of separate microlensing, nova and variable star catalogues of the central bulge region of M31. These catalogues will include a full and robust completeness analysis of the data-set and ADAP. These will in turn, allow us to begin addressing the main science goals of the project.

\section{References}

[1] Kerins E., Darnley M. J., Duke J. P., Gould A., Han C., Jeon Y.-B., Newsam A., Park B.-G., 2006, MNRAS, 365, 1099 [astro-ph/ 0502545 ]

[2] Steele I. A., et al., 2004, SPIE, 5489, 679

[3] Chung S.-J., et al., 2006, ApJ, 650, 432 [astro-ph / 0509622 ]

[4] Kim D., et al., 2007, ApJ, 666, 236 [astro-ph/0701588]

[5] Udalski A., 2003, AcA, 53, 291 [astro-ph/0401123]

[6] Alard C., 2000, A\&AS, 144, 363

[7] Cash W., 1979, ApJ, 228, 939

[8] Darnley M. J., et al., 2007, ApJ, 661, L45 [astro-ph/ 0612704 ]

[9] Calchi Novati S., Jetzer P., Scarpetta G., Giraud-Héraud Y., Kaplan J., Paulin-Henriksson S., Gould A., 2003, A\&A, 405, 851 [astro-ph/ 0304485 ]

[10] Darnley M. J., Kerins E., Newsam A. M., 2007, ATel, 1192, 1 